\section{Self-management of warfarin}

Patients receiving warfarin require regular monitoring of the international normalized ratio (INR) and appropriate dosage adjustments in order to remain within the drug's narrow therapeutic range. Although anticoagulation clinics are available in some countries, warfarin management is usually conducted by the primary care physician. Self-management (SM) of warfarin by the patient may be a superior strategy, in terms of both convenience and anticoagulation control. Sunderji and colleagues have explored this in a recent Canadian study.

The 8-month trial randomized patients to their usual physician-management (PM) or to SM ( $n=70$ in each group). Following training, those in the SM group began testing their INR with a point-of-care monitor, which calculates the INR using blood from fingertip puncture. The patients then adjusted their warfarin doses using a nomogram.

Thirteen patients dropped out of the SM group as they were unable to self-manage. Based on an intention-to-treat analysis, patients in the SM group maintained their INR values within the target range $71.8 \%$ of the time, compared with $63.2 \%$ in the PM group. This difference did not reach statistical significance. The proportion of time spent below the therapeutic range, however, was significantly lower in self-managed patients $(15.0 \%$ vs $27.3 \%, P=0.04)$. All patients completing SM preferred to continue managing their own therapy.

The authors conclude that SM was feasible and appeared safe in these patients, although maintenance of therapeutic anticoagulation was not improved. Cost of training and pointof-care monitors may be a barrier to selfmanagement strategies.

Original article Sunderji R et al. (2004) A randomized trial of patient self-managed versus physician-managed oral anticoagulation. Can J Cardiol 20: 1117-1123

\section{New approach to programmed ventricular stimulation}

Programmed ventricular stimulation is used to identify patients at high risk of sudden death following myocardial infarction. This procedure is costly in terms of personnel and equipment, however, and is not routinely available in Third
World countries. A recent study from Venezuela describes a bedside ventricular stimulation protocol for cardiac risk stratification.

Authors Fuenmayor et al. studied 412 patients with acute myocardial infarction. A subset of 50 patients had a low left-ventricular ejection fraction $(<40 \%)$ and ventricular arrhythmia, late potentials or low heart-rate variability. These patients were considered to be at high risk of sudden death and were subjected to the ventricular stimulation protocol. Under fluoroscopy guidance and electrocardiographic monitoring, a central venous access was placed and a quadripolar catheter was advanced to the right-ventricular apex. Using a portable stimulator, programmed ventricular stimulation was then performed, with up to three extrastimuli.

No complications were attributable to the procedure. Reproducible ventricular tachycardia or ventricular fibrillation was induced in six patients. These patients received amiodarone and an automatic implantable cardioverter defibrillator was subsequently placed in five cases. During a mean follow-up of $22 \pm 6$ months, all five patients received appropriate discharges from the device. All 50 high-risk patients were alive at the end of the study.

The authors conclude that bedside programmed stimulation was safe and effective in this study. Since the procedure is cheaper and more convenient than conventional methods, they suggest that it deserves further investigation as an alternative means of risk stratification in patients with myocardial infarction.

Original article Fuenmayor AJ et al. (2004) Bedside programmed ventricular stimulation for sudden death risk stratification. Int J Cardiol 97: 69-72

\section{Ultrasound diagnosis of recurrent deep vein thrombosis}

Although compressibility of the venous lumen indicates no thrombus is present, noncompressibility may not indicate the reverse. Instead, it may be a sign of previous thrombosis with persistent abnormal ultrasound data. Determination of thrombus recurrence is, therefore, difficult to prove using current methods. Linkins et al. have investigated whether an increase in thrombus length could serve as a surrogate indicator of thrombus recurrence. 